\title{
Randomized controlled trial of a minimal versus extended Internet-based intervention for problem drinkers: study protocol
}

\author{
John A Cunningham ${ }^{1,2^{*}}$, Christian S Hendershot ${ }^{3,4}$ and Jürgen Rehm ${ }^{1,4,5,6}$
}

\begin{abstract}
Background: Problem drinking causes great harm to the person and to society. Most problem drinkers will never seek treatment. The current trial will test the efficacy of two Internet interventions for problem drinking - one minimal and the other extended - as an alternate means of providing help to those in need.

Methods/Design: A double blinded, four-wave panel design with random assignment to two experimental conditions will be used in this study. Participants will be recruited through a comprehensive recruitment strategy consisting of online and print advertisements asking for people who are 'interested in helping us develop and evaluate Internet-based interventions for problem drinkers.' Potential participants will be screened to select problem drinkers who have home access to the Internet. Participants will be sent to a password-protected Internet site and, upon signing in, will be randomized to be provided access to the minimal or extended Internet-based intervention. Six-month, twelve-month, and two-year drinking outcomes will be compared between experimental conditions. The primary hypothesis is that participants in the extended Internet intervention condition will display significantly improved drinking outcomes at twelve months compared to participants in the minimal intervention.
\end{abstract}

Discussion: The findings of this trial will contribute to the growing literature on Internet interventions for problem drinkers. In addition, findings from this trial will contribute to the scarce literature available evaluating the long-term efficacy of brief interventions for alcohol problems.

Trial registration: Clinical Trials.gov \#NCT01874509; First submitted June 17, 2013.

\section{Background}

Hazardous alcohol use is the second leading contributor to the preventable burden of disease in high income countries [1-4]. In 2001, 6\% of all deaths in Canadian adults under the age of 70 were attributable to alcohol [3]. In addition, the economic costs of problem drinking are also high. In 2002, it was estimated that alcoholrelated health care cost Canadians $\$ 2.3$ billion dollars [5] and there are also other substantial economic costs, mainly attributed to lost productivity and law enforcement [4]. Alcohol use is thus a key factor in population health in Canada.

\footnotetext{
* Correspondence: john.cunningham@anu.edu.au

'Department of Social and Epidemiological Research, Centre for Addiction and Mental Health, Toronto, Canada

${ }^{2}$ National Institute for Mental Health Research, Australian National University, Canberra, Australia

Full list of author information is available at the end of the article
}

There are many more problem drinkers than those with alcohol dependence [6]. The majority of these problem drinkers will never seek treatment [7-10]. Nevertheless, studies assessing the level of interest in self-help materials for problem drinkers in the general population reveal that a considerable number of drinkers, especially heavier drinkers, would like to receive aids to help them drink less [11]. Koski-Jännes and Cunningham [12] found that $39 \%$ of current drinkers were interested in computerized summaries comparing their drinking to that of other Canadians, and that fully $70 \%$ of problem drinkers were interested in receiving such a self-help intervention.

The widespread accessibility of the Internet may prove it an ideal vehicle for the delivery of self-help material to problem drinkers. A recent systematic review [13] emphasized the fast growing body of evidence for the evidence supporting Internet-based Interventions (IBIs). 
The majority of this research suggests that personalized feedback interventions can reduce drinking over short intervals [14-17]. However, there is little research evaluating the efficacy of more comprehensive web-based interventions for facilitating long-term changes in drinking [18]. This limitation is notable for at least two reasons. First, initial changes in alcohol use are most often not sustained $[19,20]$; and the strategies needed to maintain long-term change may be different than those required for initial change [21-23]. Whereas personalized feedback could provide a source of initial motivation to reduce drinking, more comprehensive cognitive-behavioural strategies may be more effective for aiding in sustained behaviour change and relapse prevention. Second, there have been increasing calls to adapt efficacious alcohol treatments to allow for sustained delivery, consistent with a continuing care model of treatment [24-26]. One advantage of IBIs is that they can be sustained indefinitely (i.e., the cost of long-term or comprehensive interventions will often be comparable to those of brief interventions).

\section{Pilot study}

A pilot trial conducted by the authors provides preliminary evidence suggesting that IBIs that incorporate cognitive behavioural strategies may be more effective than those that provide personalized normative feedback. The pilot trial specifically assessed whether six-month outcomes varied between problem drinkers provided with minimal IBIs (i.e. brief normative personalized feedback) versus extended IBIs that include comprehensive cognitivebehavioural strategies. In this trial, participants were randomized into either an extended intervention (i.e. Alcohol Help Centre: AHC) or a minimal intervention (i.e. Check Your Drinking: CYD). The AHC is a website that contains a series of cognitive-behavioural and relapse prevention tools that have been found to be effective in promoting reductions in drinking in clinical settings, whereas the CYD is a brief screener designed to provide personalized normative feedback aimed at motivating reductions in drinking. Some evidence was found for the added impact of the AHC extended intervention above that observed on the CYD minimal intervention [27]. There were, however, several limitations of this pilot trial that will be addressed in the present study. First, the present study will employ a double blind design to protect against other sources of bias. Second, a long-term follow-up will be included to test the hypothesis that intervention-related reductions in drinking are sustained to a greater extent in the context of a more comprehensive intervention.

\section{Hypotheses}

\section{Primary hypothesis}

Participants in the extended Internet intervention condition will display significantly improved drinking outcomes at twelve months compared to participants in the minimal Internet intervention condition.

Secondary Hypotheses:

Secondary Hypothesis 1: Participants in the extended Internet intervention condition will display significantly improved drinking outcomes at six months compared to participants in the minimal Internet intervention condition.

Secondary Hypothesis 2: Participants in the extended Internet intervention condition will display significantly improved drinking outcomes at two years compared to participants in the minimal Internet intervention condition.

Secondary Hypothesis 3: Participants in the extended Internet intervention conditions will display significantly improved health-related quality of life at twelve months compared to participants in the minimal Internet intervention condition.

Secondary Hypothesis 4: Participants in the extended Internet intervention condition who have more involvement with the $\mathrm{AHC}$ intervention between baseline and twelve-month follow-up will demonstrate more improvement in drinking outcomes at twelvemonth follow-up, compared to respondents in the extended Internet intervention condition who have less involvement with the $\mathrm{AHC}$ intervention.

\section{Methods/Design \\ Design}

This is a double-blind, four-wave panel randomized control trial of two IBIs for alcohol problems, with three follow-up periods (6 months, 12 months and 2 years). A comprehensive recruitment strategy using online and print advertisements will be used to recruit current drinkers interested in helping researchers "revise and evaluate Internet-based interventions for alcohol users." Interested potential participants will be directed to complete an online consent form and a baseline questionnaire. Those found to be eligible based on their responses to the baseline questionnaire will be provided with a unique password to the study website. Upon accessing the website, they will be randomized into one of the two interventions: the minimal Internet-based Check Your Drinking personalized feedback intervention or the extended Internet-based Alcohol Help Center. Only participants who use their password and access the website will be included in the trial. Participants will be provided an honorarium of $\$ 20$ for completing each follow-up questionnaire and an honorarium of $\$ 10$ for initially accessing the study website (the latter will be employed in order to avoid loss of potential participants at this point). Potential participants deemed ineligible to participate will be compensated $\$ 20$ for having completed the 
baseline questionnaire. See Figure 1 for a Consort Diagram summarizing this trial design.

\section{Ethical approval}

The research methods to be used in this study have been approved by the standing ethics review committee of the Centre for Addiction and Mental Health.

\section{Participants - inclusion and exclusion criteria}

Participation in the trial will be restricted to those who are 19 and above (legal drinking age in Canada), current problem drinkers (as indicated by a score of 8 or more on the Alcohol Use Disorder Identification Test AUDIT) $[28,29]$, have home access to the Internet and read English (the AHC is currently only available in
English). It should be noted that this recruitment procedure may result in the participation of some individuals who are dependent on alcohol. However, as Heather p. 366, [30] has well summarized, "evidence shows that brief interventions are effective and should be used for individuals who are not actively seeking help at specialist agencies. This justification is again independent of level of seriousness, although most recipients of communitybased interventions will obviously have problems of a less severe variety." Indeed, as respondents in this trial will not be treatment seekers, the IBIs could easily serve as their first exposure to any services for alcohol problems. Thus, it is appropriate to include all eligible individuals in this evaluation, no matter the severity of their problems. Further it is also possible that some respondents

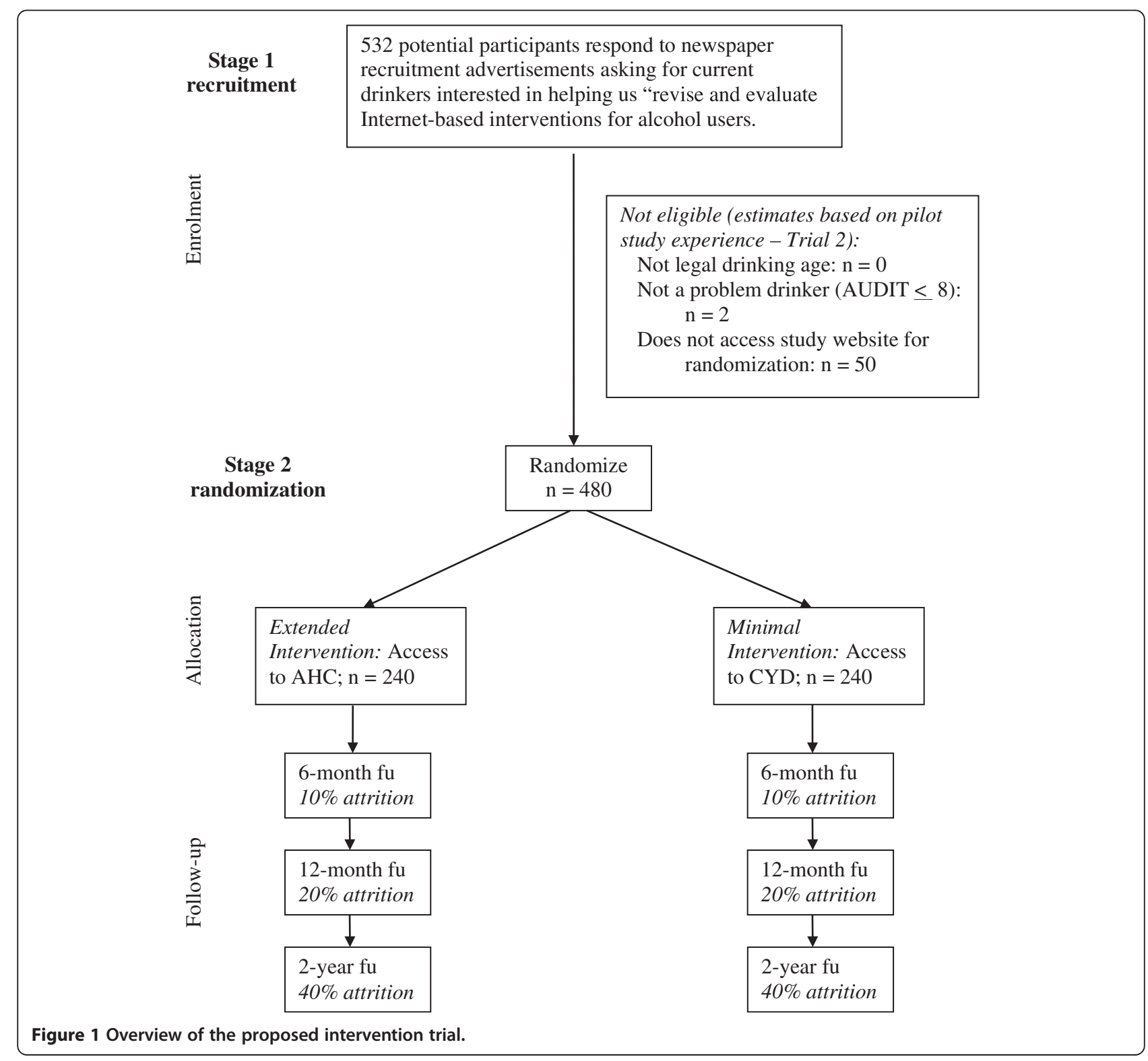


will have accessed addictions treatment or other forms of assistance for alcohol problems (such as Internet-based interventions) at some point in their lives. This will be assessed on the baseline questionnaire, however respondents will not be excluded from the study due to prior treatment access because the intent is to evaluate the impact of the interventions in the extended range of potential community participants. However, random assignment to condition should ensure that socio-demographic characteristics, such as treatment access, will be randomly assigned across conditions.

\section{Interventions}

\section{Minimal intervention experimental condition}

A randomized half of the subjects meeting eligibility criteria will be assigned to the Check Your Drinking screener (CYD; available at www.CheckYourDrinking. net). The CYD screener consists of an 18 item screener, the responses from which are then used to generate a personalized feedback Final Report containing normative feedback content as well as a summary of the amount and risks associated with the recipient's drinking [31,32].

\section{Extended internet-based intervention experimental condition}

Subjects randomly assigned to the extended internetbased intervention will be directed to the Alcohol Help Centre (AHC; located at www.AlcoholHelpCentre.net). The AHC contains cognitive behavioral, motivational and relapse prevention components. In addition, there is an online support group moderated by health educators [33]. More details of the AHC are provided elsewhere [27].

\section{Randomization}

After providing electronic consent, a letter will be sent to each participant, thanking them for agreeing to participate in the study. Contained in this letter will be a World Wide Web address unique to the study and a password unique to each participant. Upon accessing the website, the participant will enter his or her password and be randomly assigned to either the minimal or the extended Internet-based intervention using a simple randomization without replacement built into the website (no stratification or minimization given the large sample size in this trial).

\section{Blinding}

Participants will be blind to their experimental condition as their password will always direct them to the same intervention after the initial randomization. Research staff involved in the trial will not be informed of respondents' group allocation during interventions or at followup. The generation of the randomized sequence and loading of it into the server database has been conducted by a staff member who will not be involved in the implementation of the trial or with any participant contact.

\section{Outcome measures}

\section{Primary outcome measure}

In order to gain a clear picture of changes in drinking status, both the quantity and frequency of alcohol consumption will be measured as well as the frequency of heavy drinking occasions $[34,35]$. Therefore, the primary outcome measure will be the AUDIT-C scale (composite measure of frequency of drinking, typical quantity of drinking on one occasion, and frequency of drinking five of more drinks on one occasion). This measure was chosen because it comprises the main predictors of risky drinking in a recent analysis of the relation of drinking to the development of alcohol dependence [36]. This measure was chosen rather than a drinking diary data collection method because summary measures generated from a drinking diary method have been shown to be functionally identical to single point retrospective measures [37] and because the inclusion of a drinking diary in research has been shown to increase attrition in studies employing mailed surveys [38].

\section{Secondary outcome measure}

Secondary outcome measures are: (1) number of drinks in a typical week [39-41]; and (2) highest number of drinks on a single occasion. Finally, Health related Quality of Live (HRQOL) will be measured at each time point using the WHOQoL-8. This short form has been used in a number of countries, is robust psychometrically, and overall performance is strongly correlated with scores from the original WHOQoL [42].

\section{Data analysis}

\section{Power analysis}

The AUDIT-C measure is employed in this power calculation because this scale is a well-validated screener that has research supporting the amount of change that is needed in order to be clinically significant. Ideally, the study should be powered to be able to detect a difference in 1 point on the AUDIT-C scale as this was the added impact of the extended intervention over the minimal intervention observed in a previous pilot study (albeit at a six-month follow-up). A reduction from 9 points to 8 points on the AUDIT-C scale is also a clinically significant effect, representing $15 \%$ fewer people drinking beyond recommended low risk guidelines and a five drink per week reduction in typical weekly consumption (findings from the 2009 CAMH Monitor, a general population survey of Ontario residents) [43]. Further, a relatively variable sample is assumed with a standard deviation of 3.0 units on the AUDIT-C (standard deviation in the pilot trial was 2.5). 
Following the convention that studies should be designed to have a statistical power of at least $80 \%$, and that hypotheses be tested at the .05 level of significance, these specifications resulted in a final sample (required after attrition) of $\mathrm{N}=286$ (143 participants per condition).

In addition, there is allowance for the possibility of a substantial loss to follow-up in order to ensure sufficient numbers for analyses. Further, while the power analysis was conducted referring to the primary hypothesis (12 months follow up), sufficient participants will be recruited in order to test for a similar effect size at the two-year time point. Specifically, there is an allowance for a $20 \%$ attrition at the one-year follow-up and $40 \%$ attrition at the time of the two-year follow-up, and proposing to recruit a total of 480 participants at baseline (thus, allowing for a possible $40 \%$ loss to follow-up at the two year time point).

\section{Analysis plan: primary hypothesis testing}

The primary hypothesis is that participants in the extended Internet intervention condition will display significantly improved drinking outcomes at twelve months compared to participants in the minimal Internet intervention condition. This will be tested for the primary outcome variable, the AUDIT-C, using an analysis of covariance with experimental condition as the between subject variable and the baseline value of the outcome variable as the covariate. A maximum likelihood approach will be used to replace any missing data at twelve-month follow-up. Sensitivity analyses will be conducted with participants lost to follow-up excluded.

\section{Secondary analyses}

To test the difference across time between the two conditions (minimal or extended Internet-based intervention), a group $(1,2) \times$ time $(0,6,12$, and 24$)$ repeated measures MANOVA will be performed, including the primary outcome variable (AUDIT-C) and the two secondary outcome drinking variables (number of drinks in a typical week and greatest number of drinks on one occasion). Missing data will be replaced using a maximum likelihood approach. Planned contrasts will determine the nature of the differences observed. These planned contrasts will be stepped such that significant differences must be observed at the six months' time point before comparisons will be made between condition at the twelve months' time point, etc. Effect sizes will be calculated to determine the magnitude of differences in standard units. Interaction terms will be added to the MANOVA to test for sex differences (i.e., is there an interaction effect of subjects' sex by experimental condition on drinking outcomes?). Finally, a separate repeatedmeasure ANOVA will be employed to test the hypothesis on the impact of receiving access to the Internet-based interventions on Health Related Quality of Life (secondary hypothesis 3 ). As part of these secondary analyses, we will also conduct a chi-square test to explore whether there is differential loss to follow-up between experimental conditions.

The proportion of each group that has sought further treatment (total and categorized into type of treatment such as support groups, residential, individual counseling) will be compared using $x^{2}$ statistics. If the proportion of treatment-seekers is high then further analyses will be conducted to explore the significance of this treatment to outcome.

\section{Discussion}

The efficacy of IBIs to reduce drinking over short intervals has received increasing empirical evidence as is indicated in several systematic reviews [13-17]. However, research demonstrating that short-term changes in alcohol consumption are often not maintained $[19,20]$ warrants an investigation of the efficacy of IBIs in long-term reductions in alcohol consumption. This project will be one of the first such trial to investigate the long-term benefits of IBIs among problem drinkers and compare the effectiveness of a normative personalized feedback intervention to one that contains a range of cognitivebehavioural and relapse prevention tools.

\section{Competing interests}

The authors declare that they have no competing interests.

\section{Authors' contributions}

All authors have made an intellectual contribution to this research trial. JAC is the principal investigator of the trial, with overall responsibility for the project. He conceived the study, prepared the protocol, and oversees all phases of the project. CSH and JR have provided leadership with respect to the design of the study as well as to the analytical approaches to be employed. All authors have provided approval for the submission of this manuscript.

\section{Acknowledgements}

This research is funded by the Canadian Institutes of Health Research (CIHR). Support to CAMH for salary of scientists and infrastructure has been provided by the Ontario Ministry of Health and Long Term Care. The views expressed in this article do not necessarily reflect those of the Ministry of Health and Long Term Care.

\section{Author details}

${ }^{1}$ Department of Social and Epidemiological Research, Centre for Addiction and Mental Health, Toronto, Canada. ${ }^{2}$ National Institute for Mental Health Research, Australian National University, Canberra, Australia. ${ }^{3}$ Campbell Family Mental Health Research Institute, Centre for Addiction and Mental Health, Toronto, Canada. ${ }^{4}$ Department of Psychiatry, University of Toronto, Toronto, Canada. ${ }^{5}$ Dalla Lana School of Population Health, University of Toronto, Toronto, Canada. ${ }^{6}$ Technische Universität, Dresden, Germany.

Received: 18 December 2014 Accepted: 2 January 2015

Published online: 21 January 2015

\section{References}

1. World Health Organization. Global health risks: Mortality and burden of disease attributable to selected major risks. Geneva, Switzerland: World Health Organization; 2009. 
2. Rehm J, Mathers C, Popova S, Thavorncharoensap M, Teerawattananon Y, Patra J. Global burden of disease and injury and economic cost attributable to alcohol use and alcohol-use disorders. Lancet. 2009;373(9682):2223-33.

3. Rehm J, Patra J, Popova S. Alcohol-attributable mortality and potential years of life lost in Canada 2001: Implications for prevention and policy. Addiction. 2006;101(3):373-84.

4. Rehm J, Gnam W, Popova S, Baliunas D, Brochu S, Fischer B, et al. The costs of alcohol, illegal drugs, and tobacco in Canada, 2002. J Stud Alcohol Drugs. 2007;68(6):886-95.

5. Taylor B, Rehm J, Patra J, Popova S, Baliunas D. Alcohol-attributable morbidity and resulting health care costs in Canada in 2002: recommendations for policy and prevention. J Stud Alcohol Drugs. 2007;68(1):36-47.

6. Institute of Medicine. Broadening the base of treatment for alcohol problems. Washington, DC: National Academy Press; 1990.

7. Cunningham JA, Breslin FC. Only one in three people with alcohol abuse or dependence ever seek treatment. Addict Behav. 2004;29(1):221-3.

8. Roizen R, Cahalan D, Shanks P. "Spontaneous remission" among untreated problem drinkers. In: Kandel DB, editor. Longitudinal research on drug use: Empirical findings and methodological issues. Washington, DC: Hemisphere; 1978. p. 197-221.

9. Hasin DS. Treatment/self-help for alcohol-related problems: Relationship to social pressure and alcohol dependence. J Stud Alcohol. 1994;55(6):660-6.

10. Burton TL, Williamson DL. Harmful effects of drinking and the use and perceived effectiveness of treatment. J Stud Alcohol. 1995;56:611-5.

11. Cunningham JA, Wild TC, Walsh GW. Interest in self-help materials in a general population sample of drinkers. Drugs Educ Prev Policy. 1999;6(2):209-13.

12. Koski-Jännes $A$, Cunningham JA. Interest in different forms of self-help in a general population sample of drinkers. Addict Behav. 2001;26:91-9.

13. Riper H, Blankers M, Hadiwijaya H, Cunningham J, Clarke S, Wiers R, et al. Effectiveness of guided and unguided low-intensity internet interventions for adult alcohol misuse: a meta-analysis. PLoS One. 2014;9(6):e99912.

14. Bewick BM, Trusler K, Barkham M, Hill AJ, Cahill J, Mulhern B. The effectiveness of web-based interventions designed to decrease alcohol consumption - A systematic review. Prev Med. 2008;47(1):17-26.

15. Tait RJ, Christensen $\mathrm{H}$. Internet-based interventions for young people with problematic substance use: a systematic review. Med J Aust. 2010;192(11 Suppl):S15-21.

16. Khadjesari Z, Murray E, Hewitt C, Hartley S, Godfrey C. Can stand-alone computer-based interventions reduce alcohol consumption? A systematic review. Addiction. 2011;106(2):267-82.

17. White A, Kavanagh D, Stallman H, Klein B, Kay-Lambkin F, Proudfoot J, et al. Online alcohol interventions: a systematic review. J Med Internet Res. 2010;12(5):e62.

18. Riper H, Spek V, Boon B, Conijn B, Kramer J, Martin-Abello K, et al. Effectiveness of E-self-help interventions for curbing adult problem drinking: a meta-analysis. J Med Internet Res. 2011;13(2):e42.

19. Brandon TH, Vidrine Jl, Litvin EB. Relapse and relapse prevention. Annu Rev Clin Psycho. 2007:3:257-84

20. Witkiewitz K. Predictors of heavy drinking during and following treatment. Psychol Addict Behav. 2011;25(3):426-38.

21. Larimer ME, Palmer RS, Marlatt GA. Relapse prevention. An overview of Marlatt's cognitive-behavioral model. Alcohol Res Health. 1999;23(2):151-60.

22. Marlatt GA, Gordon JR. Relapse prevention. New York: Guilford Press; 1985.

23. Hendershot CS, Witkiewitz K, George WH, Marlatt GA. Relapse prevention for addictive behaviors. Subst Abuse Treat Prev Policy. 2011;6:17.

24. McKay JR, Carise D, Dennis ML, Dupont R, Humphreys K, Kemp J, et al. Extending the benefits of addiction treatment: practical strategies for continuing care and recovery. J Subst Abuse Treat. 2009;36(2):127-30.

25. McKay JR, Carise D. State of the science: Extending the benefits of addiction treatment. J Subst Abuse Treat. 2009;36(2):172-3.

26. McLellan AT. Have we evaluated addiction treatment correctly? Implications from a chronic care perspective. Addiction. 2002;97(3):249-52.

27. Cunningham JA. Comparison of two internet-based interventions for problem drinkers: randomized controlled trial. J Med Internet Res. 2012;14(4):e107.

28. Babor TF, De La Fuente MF, Saunders JB, Grant M. AUDIT - The alcohol use disorders identification test: Guidelines for use in primary health care. Geneva, Switzerland: World Health Organization; 1989.

29. Saunders JB, Aasland OG, Babor TF, De La Fuente JR, Grant M. Development of the Alcohol Use Disorders Identification Test (AUDIT): WHO collaborative project on early detection of persons with harmful alcohol consumption- 1 . Addiction. 1993;88:791-804.

30. Heather N. Psychology and brief interventions. Br J Addict. 1989;84:357-70.

31. Cunningham JA, Humpheys K, Kypri K, van Mierlo T. Formative evaluation and three-month follow-up of an online personalized assessment feedback intervention for problem drinkers. J Med Internet Res. 2006;8(2):e5.

32. Cunningham JA, Wild TC, Cordingley J, van Mierlo T, Humphreys K. A randomized controlled trial of an internet-based intervention for alcohol abusers. Addiction. 2009;104(12):2023-32.

33. Cunningham JA, van Mierlo T, Fournier R. An online support group for problem drinkers: AlcoholHelpCenter.net. Patient Educ Couns. 2008;70 (2):193-8.

34. Gmel G, Lokosha O. Self-reported frequency of drinking assessed with a closed- or open-ended question format: A split-sample study in Switzerland. J Stud Alcohol. 2000;61:450-4.

35. National Institute on Alcohol Abuse and Alcoholism. Assessing alcohol problems: A guide for clinicians and researchers. 2nd ed. Rockville, MD: National Institute on Alcohol Abuse and Alcoholism; 2005.

36. Dawson DA, Grant BF, Li TK. Quantifying the risks associated with exceeding recommended drinking limits. Alcohol Clin Exp Res. 2005;29(5):902-8.

37. Sobell LC, Agrawal S, Sobell MB, Leo Gl, Young LJ, Cunningham JA, et al. Comparison of a quick drinking screen with the timeline followback for individuals with alcohol problems. J Stud Alcohol. 2003;64(6):858-61.

38. Cunningham JA, Ansara D, Wild TC, Toneatto T, Koski-Jannes A. What is the price of perfection? The hidden costs of using detailed assessment instruments to measure alcohol consumption. J Stud Alcohol. 1999;60(6):756-8.

39. Romelsjö A, Leifman H, Nyström S. A comparative study of two methods for the measurement of alcohol consumption in the general population. Int J Epidemiol. 1995;24:929-36.

40. Kühlhorn $\mathrm{E}$, Leifman $\mathrm{H}$. Alcohol surveys with high and low coverage rate: A comparative analysis of survey strategies in the alcohol field. J Stud Alcohol. 1993;54:542-4

41. Neighbors C, Lewis MA, Atkins DC, Jensen MM, Walter T, Fossos N, et al. Efficacy of web-based personalized normative feedback: a two-year randomized controlled trial. J Consult Clin Psychol. 2010;78(6):898-911.

42. Schmidt S, Muhlan H, Power M. The EUROHIS-QOL 8-item index: psychometric results of a cross-cultural field study. Eur J Pub Health. 2006;16(4):420-8.

43. Ialomiteanu A, Adlaf EM. CAMH Monitor 2009: Technical Guide. In. Toronto: Centre for Addiction and Mental Health; 2010.

\section{Submit your next manuscript to BioMed Central and take full advantage of:}

- Convenient online submission

- Thorough peer review

- No space constraints or color figure charges

- Immediate publication on acceptance

- Inclusion in PubMed, CAS, Scopus and Google Scholar

- Research which is freely available for redistribution

Submit your manuscript at www.biomedcentral.com/submit
C) Biomed Central 